\title{
Closing the Brief Case: Bartonella henselae Endocarditis-a Case of Delayed Diagnosis
}

\author{
Kyle G. Rodino, ${ }^{a}$ Eric Stone, ${ }^{\text {b }}$ Omar Abu Saleh, ${ }^{\mathrm{b}}$ Elitza S. Theel ${ }^{\mathrm{a}}$
}

aDivision of Clinical Microbiology, Department of Laboratory Medicine and Pathology, Mayo Clinic, Rochester, Minnesota, USA

bDivision of Infectious Diseases, Mayo Clinic, Rochester, Minnesota, USA

KEYWORDS Bartonella, infective endocarditis

\section{ANSWERS TO SELF-ASSESSMENT QUESTIONS}

1. Which of the following is the most sensitive noninvasive microbiologic assay for detection of suspected Bartonella spp. endocarditis?
a. Blood culture
b. Culture of heart valve tissue
c. Bartonella sp. IgG-specific serologic testing
d. Broad-range $16 \mathrm{~S}$ PCR and sequencing from heart valve tissue

Answer: c. Serologic testing for antibodies to Bartonella spp., particularly IgGclass antibodies, remains the most sensitive, noninvasive means by which to make a diagnosis of Bartonella endocarditis. Nucleic acid amplification tests (NAATs) on blood or blood fractions are notoriously insensitive and would only be preferred on explanted heart valve tissue.

2. A 63-year-old male originally from Brazil and now living in a homeless shelter presents to the emergency department with a fever, headache, rash, and bone pain in the shins, neck, and back. On examination, lice are visible on the patient's clothes and hospital bed sheets. Which of the following is the most likely causative organism?
a. Bartonella henselae
b. Bartonella quintana
c. Bartonella bacilliformis
d. Bartonella alsatica

Answer: b. B. quintana is transmitted by lice and is the most frequent Bartonella sp. associated with infection in homeless individuals, given the limited opportunities for routine hygiene and frequency of close living situations.

3. Antibodies to which of the following pathogens can lead to cross-reactivity on serologic assays for detection of Bartonella sp. antibodies?
a. Anaplasma phagocytophilum
b. Brucella abortus
c. Coxiella burnetii
d. Treponema pallidum

Answer: c. Among these options, antibodies to C. burnetii may lead to falsepositive results on serologic assays for Bartonella spp. and vice versa. Crossreactivity with Chlamydophila pneumoniae and some spotted fever group Rickettsia has also been reported.

Citation Rodino KG, Stone E, Abu Saleh O, Theel ES. 2019. Closing the Brief Case: Bartonella henselae endocarditis - a case of delayed diagnosis. J Clin Microbiol 57:e00123-19. https:// doi.org/10.1128/JCM.00123-19. Editor Carey-Ann D. Burnham, Washington University School of Medicine Copyright $\odot 2019$ American Society for Microbiology. All Rights Reserved. Address correspondence to Elitza S. Theel, theel.elitza@mayo.edu.

K.G.R. and E.S. contributed equally to this work. See https://doi.org/10.1128/JCM.00114-19 in this issue for case presentation and discussion. Published 26 August 2019 


\section{TAKE-HOME POINTS}

- Bartonella sp. endocarditis is the second most common cause of culturenegative endocarditis following $C$. burnetii.

- Diagnosis of Bartonella sp. endocarditis is most frequently established by serologic testing. Molecular assays are insensitive in blood or blood fractions, and culture is not routinely recommended for this pathogen.

- A significant percentage of individuals may be seropositive for lgG antibodies to $B$. henselae at titers of $\leq 1: 128$, and therefore diagnosis of a current $B$. henselae infection should be established based on titers of $\geq 1: 256$ and/or on demonstrating a 4-fold rise in IgG antibody titers between acute and convalescent sera. 